Proyecciones

Vol. 21, No 3, pp. 277-289, December 2002.

Universidad Católica del Norte

Antofagasta - Chile

\title{
JORDAN NILALGEBRAS OF DIMENSION 6
}

\author{
LUISA ELGUETA * \\ and \\ AVELINO SUAZO \\ Universidad de La Serena, Chile
}

\begin{abstract}
It is known the classification of commutative power-associative nilalgebras of dimension $\leq 4$ (see, [4]). In [2], we give a description of commutative power-associative nilalgebras of dimension 5 . In this work we describe Jordan nilalgebras of dimension 6.
\end{abstract}

AMS Subject Classification : 17 C 10 .

${ }^{*}$ Research supported by Conicyt-Chile, Proyecto Fondecyt-Líneas Complementarias 8990001 . 


\section{Preliminaries}

Let $A$ be a commutative algebra over a field $K$. If $x$ is an element of $A$, we define $x^{1}=x$ and $x^{k+1}=x^{k} x$ for all $k \geq 1$.

$A$ is called power-associative, if the subalgebra of $A$ generated by any element $x \in A$ is associative. An element $x \in A$ is called nilpotent, if there is an integer $r \geq 1$ such that $x^{r}=0$. If any element in $A$ is nilpotent, then $A$ is called a nilalgebra. Now $A$ is called a nilalgebra of nilindex $n \geq 2$, if $y^{n}=0$ for all $y \in A$ and there is $x \in A$ such that $x^{n-1} \neq 0$.

If $B, D$ are subspaces of $A$, then $B D$ is the subspace of $A$ spanned by all products $b d$ with $b$ in $B, d$ in $D$. Also we define $B^{1}=B$ and $B^{k+1}=B^{k} B$ for all $k \geq 1$. If there exists an integer $n \geq 2$ such that $B^{n}=0$ and $B^{n-1} \neq 0$, then $B$ is nilpotent of index $n$.

$A$ is a Jordan algebra, if it satisfies the Jordan identity $x^{2}(y x)=\left(x^{2} y\right) x$ for all $x, y$ in $A$. It is known that any Jordan algebra is power-associative, and also that any finite-dimensional Jordan nilalgebra (of characteristic $\neq 2$ ) is nilpotent (see, [5]).

We will use the following result which we give in [2] :

Proposition 1.1 If $A$ is a Jordan nilalgebra of nilindex $n \geq 3$ with $\operatorname{dim}_{K}(A)=m \geq n$, then $n-2 \leq \operatorname{dim}_{K}\left(A^{2}\right) \leq m-2$.

Throughout, $A$ will denote a commutative nilalgebra of nilindex $n \geq 3$ over a field $K$ of characteristic $\neq 2,3$. We will denote by $\left\langle x_{1}, \ldots, x_{j}>_{K}\right.$ the subspace generated over $K$ by the elements $x_{1}, \ldots, x_{j}$ in $A$. Also we will denote by $\alpha, \beta, \ldots$,etc., the elements of field $K$. If $x \in A$ with $x^{n-1} \neq 0$, then we will denote by $X$ the subspace $<x, x^{2}, \ldots, x^{n-1}>_{K}$. It is clear that $x, x^{2}, \ldots, x^{n-1}$ are linearly independent and so $\operatorname{dim}_{K}\left(A^{2}\right) \geq n-2$ and $\operatorname{dim}_{K}\left(A^{3}\right) \geq n-3$.

\section{COMMUTATIVE NILALGEBRAS OF NILINDEX 3 AND DIMENSION 6}

In this section, $A$ will denote a commutative nilalgebra of nilindex 3 . It is well known that a commutative nilalgebra of nilindex 3 is a Jordan algebra (see [6], page 114).

Since $x^{3}=0$ for all $x A$, then by linearization method we obtain that the following identities are valid in $A$ :

$$
x^{2} y+2(x y) x=0,(x y) z+(y z) x+(z x) y=0
$$


It is clear that the identity $x^{4}=\left(x^{2}\right)^{2}=0$ is valid in $A$, which implies that for all $x, y, z$ in $A$ we have :

$$
x^{2}(y x)=\left(x^{2} y\right) x=0,2(x y)^{2}+x^{2} y^{2}=0
$$

Lemma 2.1 If $\left(A^{2}\right)^{2} \neq 0$, then $\operatorname{dim}_{K}(A) \geq 8$.

Proof. If $\left(A^{2}\right)^{2} \neq 0$, then there exist $x, y \in A$ such that $x^{2} y^{2} \neq 0$. We note first that using (1) and (2), we obtain that: $x^{2}\left(y x^{2}\right)=-2\left(x\left(y x^{2}\right) x=0\right.$, $x^{2}\left(x y^{2}\right)=0, x^{2}\left(x^{2} y^{2}\right)=-2\left(\left(x^{2} y^{2}\right) x\right) x=0, x^{2} y^{2}+2\left(y^{2} x\right) x=0$ and $x^{2} y^{2}+2\left(x^{2} y\right) y=0$. We will prove that the elements $y, x, x^{2}, y^{2}, y x^{2}$, $x y^{2}, x y, x^{2} y^{2}$ are linearly independent. Let $\alpha_{1} y+\alpha_{2} x+\alpha_{3} x^{2}+\alpha_{4} y^{2}+$ $\alpha_{5} y x^{2}+\alpha_{6} x y^{2}+\alpha_{7} x y+\alpha_{8} x^{2} y^{2}=0$. Multiplying by $x^{2}$ we obtain that $\alpha_{1} y x^{2}+\alpha_{4} x^{2} y^{2}=0$. Thus $0=2 y\left(\alpha_{1} y x^{2}+\alpha_{4} x^{2} y^{2}\right)=2 \alpha_{1} y\left(y x^{2}\right)=-\alpha_{1} x^{2} y^{2}$ implies $\alpha_{1}=0$. Clearly also $\alpha_{4}=0$. Similarly we prove that $\alpha_{2}=\alpha_{3}=0$. Now we have that $\alpha_{5} y x^{2}+\alpha_{6} x y^{2}+\alpha_{7} x y+\alpha_{8} x^{2} y^{2}=0$. Multiplying by $x$ we get $\alpha_{6} x\left(x y^{2}\right)+\alpha_{7} x(x y)=0$. Hence $0=2 y\left(\alpha_{6} x\left(x y^{2}\right)+\alpha_{7} x(x y)\right)=$ $-\alpha_{6} y\left(x^{2} y^{2}\right)-\alpha_{7} y\left(y x^{2}\right)=\frac{1}{2} \alpha_{7} x^{2} y^{2}$ which implies $\alpha_{7}=0$. Finally it is clear that $\alpha_{6}=0$, and also that $\alpha_{5} y x^{2}+\alpha_{8} x^{2} y^{2}=0$ implies $\alpha_{5}=\alpha_{8}=0$. This proves what we wanted.

Lemma 2.2 If $A^{4} \neq 0$, then $\operatorname{dim}_{K}(A) \geq 7$.

Proof. By Lemma 2.1, we can suppose that $\left(A^{2}\right)^{2}=0$. Since $A^{4} \neq$ 0 , there exist elements $y, x, z$ in $A$ such that $z\left(y x^{2}\right) \neq 0$. Now using relation (1), we obtain that $2 z((y x) x)=-z\left(y x^{2}\right) \neq 0$. We will prove that $y, x, z, y x^{2}, y x, x^{2}, z\left(y x^{2}\right)$ are linearly independent. Let $(\mathbf{1}): \alpha_{1} y+$ $\alpha_{2} x+\alpha_{3} z+\alpha_{4} y x^{2}+\alpha_{5} y x+\alpha_{6} x^{2}+\alpha_{7} z\left(y x^{2}\right)=0$. Multiplying by $y x^{2}$ we get $0=\alpha_{1} y\left(y x^{2}\right)+\alpha_{3} z\left(y x^{2}\right)=-\frac{1}{2} \alpha_{1} y^{2} x^{2}+\alpha_{3} z\left(y x^{2}\right)=\alpha_{3} z\left(y x^{2}\right)=0$ which implies $\alpha_{3}=0$. Multiplying (1) by $x^{2}$ we obtain $\alpha_{1}=0$. We note that using (1) we get $x\left(z\left(y x^{2}\right)\right)=-z\left(x\left(y x^{2}\right)\right)-\left(y x^{2}\right)(x z)=0$ and $y\left(z\left(y x^{2}\right)\right)=-z\left(y\left(y x^{2}\right)\right)-\left(y x^{2}\right)(y z)=0$. Similarly $z\left(z\left(y x^{2}\right)\right)=0$. Now multiplying (1) by $2 x$ we obtain $0=2 \alpha_{2} x^{2}+2 \alpha_{5} x(y x)=2 \alpha_{2} x^{2}-\alpha_{5} y x^{2}$. So $0=y\left(2 \alpha_{2} x^{2}-\alpha_{5} y x^{2}\right)=2 \alpha_{2} y x^{2}-\alpha_{5} y\left(y x^{2}\right)=2 \alpha_{2} y x^{2}+\frac{1}{2} \alpha_{5} y^{2} x^{2}=2 \alpha_{2} y x^{2}$ implies $\alpha_{2}=0$. It is clear that also $\alpha_{5}=0$. Finally it is possible to prove that $\alpha_{4} y x^{2}+\alpha_{6} x^{2}+\alpha_{7} z\left(y x^{2}\right)=0$ implies $\alpha_{4}=\alpha_{6}=\alpha_{7}=0$. Therefore we conclude that $\operatorname{dim}_{K}(A) \geq 7$, as desired.

We see that Lemmas 2.1 and 2.2 imply the following result: 
Corollary 2.3 If $\operatorname{dim}_{K}(A) \leq 6$, then $\left(A^{2}\right)^{2}=A^{4}=0$.

Now if $A^{3} \neq 0$, then there exist elements $y, x$ in $A$ such that $y x^{2} \neq 0$. In this case it is easy to prove that $y, x, y x^{2}, x^{2}, y x$ are linearly independent. Therefore we obtain the following result:

Lemma 2.4 If $A^{3} \neq 0$, then $\operatorname{dim}_{K}(A) \geq 5$.

We observe that when $\operatorname{dim}_{K}(A)=6$, then by Proposition 1.1 we have that $1 \leq \operatorname{dim}_{K}\left(A^{2}\right) \leq 4$. Moreover, if $A^{3} \neq 0$ and $\operatorname{dim}_{K}(A)=6$, then $3 \leq \operatorname{dim}_{K}(A) \leq 4$.

Proposition 2.5 If $\operatorname{dim}_{K}(A)=6, A^{3} \neq 0$ and $\operatorname{dim}_{K}\left(A^{2}\right)=4$, then there exists a basis $\left\{u_{1}, u_{2}, u_{3}, u_{4}, u_{5}, u_{6}\right\}$ of $A$ such that $u_{1}^{2}=u_{6}, u_{1} u_{2}=u_{4}$, $u_{1} u_{5}=u_{3}, u_{2}^{2}=u_{5}, u_{2} u_{4}=-\frac{1}{2} u_{3}$, all other products being zero.

Proof. We know that $\left(A^{2}\right)^{2}=A^{4}=0$. Since $A^{3} \neq 0$, then there exist $y$, $x \in A$ such that $y, x, y x^{2}, y x, x^{2}$ are linearly independent. Clearly $y, x$ are not elements in $A^{2}$, and thus there exists $z \in A$ such that $\left\{y, x, y x^{2}\right.$, $\left.y x, x^{2}, z^{2}\right\}$ is a basis of $A$. As $z=\alpha_{1} y+\alpha_{2} x+\alpha_{3} y x^{2}+\alpha_{4} y x+\alpha_{5} x^{2}+$ $\alpha_{6} z^{2}$, then $z^{2}=\left(z-\alpha_{6} z^{2}\right)^{2} \in<y^{2}, x^{2}, y x, y^{2} x, y x^{2}>_{K}$. From this we see that if $y^{2} \in<x^{2}, y x, y x^{2}>_{K}$, then $z^{2} \in<x^{2}, y x, y x^{2}>_{K}$, which is a contradiction. Hence $y^{2} \notin<x^{2}, y x, y x^{2}>_{K}$, and so $\left\{y, x, y x^{2}, y x, x^{2}, y^{2}\right\}$ is a basis of $A$. Since $x y^{2} \in A^{2}$, then $x y^{2}=\alpha y x^{2}+\beta y x+\gamma x^{2}+\delta y^{2}$. Multiplying by $2 x$ we get $2 \beta x(y x)+2 \delta x y^{2}=-\beta y x^{2}+2 \delta x y^{2}=0$. Thus $-\beta y x^{2}+2 \delta\left(\alpha y x^{2}+\beta y x+\gamma x^{2}+\delta y^{2}\right)=0$, implies $\beta=\delta=0$, and so $x y^{2}=\alpha y x^{2}+\gamma x^{2}$. But $0=y\left(x y^{2}\right)=y\left(\alpha y x^{2}+\gamma x^{2}\right)=\gamma y x^{2}$ implies $\gamma=0$, and therefore $x y^{2}=\alpha y x^{2}$. Finally, if we define $u_{1}=y+\alpha x, u_{2}=x$, $u_{3}=y x^{2}, u_{4}=y x+\alpha x^{2}, u_{5}=x^{2}, u_{6}=y^{2}+2 \alpha y x+\alpha^{2} x^{2}$, we get $u_{1}^{2}=u_{6}$, $u_{1} u_{2}=u_{4}, u_{1} u_{5}=u_{3}, u_{2}^{2}=u_{5}, u_{2} u_{4}=-\frac{1}{2} u_{3}$, all other products zero.

Proposition 2.6 If $\operatorname{dim}_{K}(A)=6, A^{3} \neq 0$ and $\operatorname{dim}_{K}\left(A^{2}\right)=3$, then there exists a basis $\left\{u_{1}, u_{2}, u_{3}, u_{4}, u_{5}, u_{6}\right\}$ of $A$ such that $u_{1} u_{2}=u_{5}, u_{1} u_{6}=u_{4}$, $u_{2}^{2}=u_{6}, u_{2} u_{3}=-\beta u_{6}, u_{2} u_{5}=-\frac{1}{2} u_{4}, u_{3}^{2}=\delta u_{4}, u_{3} u_{5}=\beta u_{4}$, all other products being zero.

Proof. We know that $\left(A^{2}\right)^{2}=A^{4}=0$. Since $A^{3} \neq 0$, there exist $y$, $x \in A$ such that $y, x, y x^{2}, y x, x^{2}$ are linearly independent, and thus there exists an element $z \in A$ such that $\left\{y, x, z, y x^{2}, y x, x^{2}\right\}$ is a basis of $A$. As $y^{2} \in A^{2}$, then $y^{2}=\sigma_{1} y x^{2}+\sigma_{2} y x+\sigma_{3} x^{2}$. If $y_{0}=y-\frac{1}{2} \sigma_{2} x-\frac{1}{2} \sigma_{1} x^{2}$ we 
obtain that $y_{0}^{2}=\left(\sigma_{3}+\frac{1}{4} \sigma_{2}^{2}\right) x^{2}$, and so $0=y_{0}^{3}=\left(\sigma_{3}+\frac{1}{4} \sigma_{2}^{2}\right) y x^{2}$ which implies $\sigma_{3}+\frac{1}{4} \sigma_{2}^{2}=0$. Thus $y_{0}^{2}=0$ and clearly $\left\{y_{0}, x, z, y_{0} x^{2}, y_{0} x, x^{2}\right\}$ is a basis of $A$. Since $z x \in A^{2}$, then $z x=\alpha_{1} y_{0} x^{2}+\alpha_{2} y_{0} x+\alpha_{3} x^{2}$. If $z_{0}=z+2 \alpha_{1} y_{0} x-\alpha_{2} y_{0}-\alpha_{3} x$, we get that $\left\{y_{0}, x, z_{0}, y_{0} x^{2}, y_{0} x, x^{2}\right\}$ is a basis of $A$ with $z_{0} x=0$. Let $y_{0} z_{0}=\beta_{1} y_{0} x^{2}+\beta_{2} y_{0} x+\beta_{3} x^{2}$. If $z_{1}=z_{0}-\beta_{1} x^{2}$, we obtain that $\left\{y_{0}, x, z_{1}, y_{0} x^{2}, y_{0} x, x^{2}\right\}$ is a basis of $A$ with $z_{1} x=0$ and $y_{0} z_{1}=\beta_{2} y_{0} x+\beta_{3} x^{2}$. Now $0=y_{0}^{2} z_{1}=-2 y_{0}\left(y_{0} z_{1}\right)=-2 y_{0}\left(\beta_{2} y_{0} x+\beta_{3} x^{2}\right)=$ $\beta_{2} y_{0}^{2} x-2 \beta_{3} y_{0} x^{2}=-2 \beta_{3} y_{0} x^{2}$ implies $\beta_{3}=0$. Therefore we can suppose that in the basis $\left\{y, x, z, y x^{2}, y x, x^{2}\right\}$ of $A$, we have $y^{2}=0, z x=0$ and $y z=\beta y x$. Let $z^{2}=\delta y x^{2}+\varepsilon y x+\theta x^{2}$. Now we have that: $z(y x)=$ $-x(z y)-y(x z)=-x(z y)=-\beta x(y x)=\frac{1}{2} \beta y x^{2}, 0=4(x z) z=-2 x z^{2}=$ $-2 x\left(\delta y x^{2}+\varepsilon y x+\theta x^{2}\right)=-2 \varepsilon x(y x)=\varepsilon y x^{2}$ implies $\varepsilon=0$, and $\theta y x^{2}=$ $y\left(\delta y x^{2}+\varepsilon y x+\theta x^{2}\right)=y z^{2}=-2(y z) z=-2 \beta(y x) z=-\beta^{2} y x^{2}$ implies $\theta=-\beta^{2}$. Thus $z^{2}=\delta y x^{2}-\beta^{2} x^{2}$. Finally, if we define: $u_{1}=y, u_{2}=x$, $u_{3}=z-\beta x, u_{4}=y x^{2}, u_{5}=y x, u_{6}=x^{2}$, we obtain that $u_{1} u_{2}=u_{5}$, $u_{1} u_{6}=u_{4}, u_{2}^{2}=u_{6}, u_{2} u_{3}=-\beta u_{6}, u_{2} u_{5}=-\frac{1}{2} u_{4}, u_{3}^{2}=\delta u_{4}, u_{3} u_{5}=\beta u_{4}$, and other products zero.

We note that when $\operatorname{dim}_{K}(A)=6$, then Proposition 1.1 implies $1 \leq$ $\operatorname{dim}_{K}\left(A^{2}\right) \leq 4$. Suppose moreover that $A^{3}=0$ and $\operatorname{dim}_{K}\left(A^{2}\right)=4$. Then there exists a subspace $A_{0}$ of $A$ such that $A=A_{0} \oplus A^{2}$. Since $\operatorname{dim}_{K}\left(A_{0}\right)=2$ and $A^{2}=A_{0}^{2}$ we conclude that $\operatorname{dim}_{K}\left(A^{2}\right) \leq 3$, a contradiction. Therefore $\operatorname{dim}_{K}(A)=6$ and $A^{3}=0$ imply $1 \leq \operatorname{dim}_{K}\left(A^{2}\right) \leq 3$.

Proposition 2.7 Suppose that $\operatorname{dim}_{K}(A)=6$, with $\operatorname{dim}_{K}\left(A^{2}\right)=3$ and $A^{3}=0$.

(a) If for all $x, y \in A$ we have that $x^{2}, y^{2}, x y$ are linearly dependent, then there exist a basis $\left\{u_{1}, u_{2}, u_{3}, u_{4}, u_{5}, u_{6}\right\}$ of $A$ such that $u_{1}^{2}=u_{4}$, $u_{1} u_{2}=\frac{1}{8} \delta^{-1} \varepsilon^{-1} u_{4}+2 \delta \varepsilon u_{5}, u_{1} u_{3}=\frac{1}{4} \delta^{-1} u_{4}+\delta u_{6}, u_{2}^{2}=u_{5}, u_{2} u_{3}=$ $\varepsilon u_{5}+\frac{1}{4} \varepsilon^{-1} u_{6}, u_{3}^{2}=u_{6}$ with $\delta \varepsilon \neq 0$, all other products zero.

(b) If there exist elements $y, x$ in $A$ such that $x^{2}, y^{2}, x y$ are linearly independent, then there exist a basis $\left\{u_{1}, u_{2}, u_{3}, u_{4}, u_{5}, u_{6}\right\}$ of $A$ such that $u_{1}^{2}=\alpha_{1} u_{4}+\beta_{1} u_{5}+\gamma_{1} u_{6}, u_{1} u_{2}=\beta u_{5}, u_{1} u_{3}=\alpha_{0} u_{4}+\beta_{0} u_{5}+\gamma_{0} u_{6}$, $u_{2}^{2}=u_{4}, u_{2} u_{3}=u_{6}, u_{3}^{2}=u_{5}$, all other products zero.

Proof. To prove $(a)$, we consider $x, y, z$ in $A$ such that $x^{2}, y^{2}, z^{2}$ are linearly independent. We will prove that $x, y, z, x^{2}, y^{2}, z^{2}$ are linearly independent. If $\delta_{1} x+\delta_{2} y+\delta_{3} z+\delta_{4} x^{2}+\delta_{5} y^{2}+\delta_{6} z^{2}=0$, then $\delta_{1} x=-\left(\delta_{2} y+\right.$ 
$\left.\delta_{3} z+\delta_{4} x^{2}+\delta_{5} y^{2}+\delta_{6} z^{2}\right)$ which implies that $\delta_{1}^{2} x^{2}=\delta_{2}^{2} y^{2}+2 \delta_{2} \delta_{3} y z+\delta_{3}^{2} z^{2}$. By hypothesis $y z \in<y^{2}, z^{2}>_{K}$, and so $\delta_{1}=0$. Similarly we prove that $\delta_{2}=\delta_{3}=0$, and clearly $\delta_{4}=\delta_{5}=\delta_{6}=0$. Therefore $\left\{x, y, z, x^{2}, y^{2}, z^{2}\right\}$ is a basis of $A$. By hypothesis $x y=\alpha x^{2}+\beta y^{2}, x z=\gamma x^{2}+\delta z^{2}, y z=\varepsilon y^{2}+\theta z^{2}$, and also for all $\alpha_{1}, \alpha_{2}, \alpha_{3}, \beta_{1}, \beta_{2}, \beta_{3}$ in $K$, the vectors $\left(\alpha_{1} x+\alpha_{2} y+\alpha_{3} z\right)^{2}$, $\left(\alpha_{1} x+\alpha_{2} y+\alpha_{3} z\right)\left(\beta_{1} x+\beta_{2} y+\beta_{3} z\right),\left(\beta_{1} x+\beta_{2} y+\beta_{3} z\right)^{2}$ are linearly dependent. We have that $\left(\alpha_{1} x+\alpha_{2} y+\alpha_{3} z\right)^{2}=\left(\alpha_{1}^{2}+2 \alpha_{1} \alpha_{2} \alpha+2 \alpha_{1} \alpha_{3} \gamma\right) x^{2}+\left(\alpha_{2}^{2}+\right.$ $\left.2 \alpha_{1} \alpha_{2} \beta+2 \alpha_{2} \alpha_{3} \varepsilon\right) y^{2}+\left(\alpha_{3}^{2}+2 \alpha_{1} \alpha_{3} \delta+2 \alpha_{2} \alpha_{3} \theta\right) z^{2},\left(\alpha_{1} x+\alpha_{2} y+\alpha_{3} z\right)\left(\beta_{1} x+\right.$ $\left.\beta_{2} y+\beta_{3} z\right)=\left(\alpha_{1} \beta_{1}+\alpha_{1} \beta_{2} \alpha+\alpha_{2} \beta_{1} \alpha+\alpha_{1} \beta_{3} \gamma+\alpha_{3} \beta_{1} \gamma\right) x^{2}+\left(\alpha_{2} \beta_{2}+\alpha_{1} \beta_{2} \beta+\right.$ $\left.\alpha_{2} \beta_{1} \beta+\alpha_{2} \beta_{3} \varepsilon+\alpha_{3} \beta_{2} \varepsilon\right) y^{2}+\left(\alpha_{3} \beta_{3}+\alpha_{1} \beta_{3} \delta+\alpha_{3} \beta_{1} \delta+\alpha_{2} \beta_{3} \theta+\alpha_{3} \beta_{2} \theta\right) z^{2}$, and $\left(\beta_{1} x+\beta_{2} y+\beta_{3} z\right)^{2}=\left(\beta_{1}^{2}+2 \beta_{1} \beta_{2} \alpha+2 \beta_{1} \beta_{3} \gamma\right) x^{2}+\left(\beta_{2}^{2}+2 \beta_{1} \beta_{2} \beta+\right.$ $\left.2 \beta_{2} \beta_{3} \varepsilon\right) y^{2}+\left(\beta_{3}^{2}+2 \beta_{1} \beta_{3} \delta+2 \beta_{2} \beta_{3} \theta\right) z^{2}$. We conclude that for all $\alpha_{1}, \alpha_{2}, \alpha_{3}$, $\beta_{1}, \beta_{2}, \beta_{3}$ in $K$, the vectors $\left(\alpha_{1}^{2}+2 \alpha_{1} \alpha_{2} \alpha+2 \alpha_{1} \alpha_{3} \gamma, \alpha_{2}^{2}+2 \alpha_{1} \alpha_{2} \beta+2 \alpha_{2} \alpha_{3} \varepsilon\right.$, $\left.\alpha_{3}^{2}+2 \alpha_{1} \alpha_{3} \delta+2 \alpha_{2} \alpha_{3} \theta\right),\left(\alpha_{1} \beta_{1}+\alpha_{1} \beta_{2} \alpha+\alpha_{2} \beta_{1} \alpha+\alpha_{1} \beta_{3} \gamma+\alpha_{3} \beta_{1} \gamma, \alpha_{2} \beta_{2}+\right.$ $\left.\alpha_{1} \beta_{2} \beta+\alpha_{2} \beta_{1} \beta+\alpha_{2} \beta_{3} \varepsilon+\alpha_{3} \beta_{2} \varepsilon, \alpha_{3} \beta_{3}+\alpha_{1} \beta_{3} \delta+\alpha_{3} \beta_{1} \delta+\alpha_{2} \beta_{3} \theta+\alpha_{3} \beta_{2} \theta\right)$, $\left(\beta_{1}^{2}+2 \beta_{1} \beta_{2} \alpha+2 \beta_{1} \beta_{3} \gamma, \beta_{2}^{2}+2 \beta_{1} \beta_{2} \beta+2 \beta_{2} \beta_{3} \varepsilon, \beta_{3}^{2}+2 \beta_{1} \beta_{3} \delta+2 \beta_{2} \beta_{3} \theta\right)$ in $K^{3}$ are linearly dependent, which implies that $\beta=2 \delta \varepsilon, \delta=2 \beta \theta, \varepsilon=2 \gamma \beta$, $\theta=2 \alpha \delta, \gamma=2 \alpha \varepsilon, \alpha=2 \gamma \theta$. We observe that if $0 \in\{\alpha, \beta, \gamma, \delta, \varepsilon, \theta\}$, then $\alpha=\beta=\gamma=\delta=\varepsilon=\theta=0$. In this case $(x+y)^{2},(x+z)^{2}$ and $(x+y)(x+z)$ are linearly independent, a contradiction. Therefore $\alpha, \beta, \gamma, \delta, \varepsilon, \theta$ are not zero and we get $\alpha=\frac{1}{8} \delta^{-1} \varepsilon^{-1}, \beta=2 \delta \varepsilon, \gamma=\frac{1}{4} \delta^{-1}, \theta=\frac{1}{4} \varepsilon^{-1}$. Finally, if we define $u_{1}=x, u_{2}=y, u_{3}=z, u_{4}=x^{2}, u_{5}=y^{2}, u_{6}=z^{2}$, we obtain $(a)$.

Suppose now that there exist $y, x$ in $A$ such that $x^{2}, y^{2}, x y$ are linearly independent. In this case it is easy to prove that $x, y, x^{2}, y^{2}, x y$ are linearly independent. Let $u$ be an element in $A$ such that $\left\{u, x, y, x^{2}, y^{2}, x y\right\}$ is a basis of $A$. Since $u x \in A^{2}$, then $u x=\alpha x^{2}+\beta y^{2}+\gamma x y$. If $u_{0}=u-\alpha x-\gamma y$, then $u_{0} x=\beta y^{2}$. Finally, if we define $u_{1}=u_{0}, u_{2}=x, u_{3}=y, u_{4}=x^{2}$, $u_{5}=y^{2}, u_{6}=x y$, we get $(b)$.

Proposition 2.8 If $\operatorname{dim}_{K}(A)=6, A^{3}=0$ and $\operatorname{dim}_{K}\left(A^{2}\right)=2$, then there exists a basis $\left\{u_{1}, u_{2}, u_{3}, u_{4}, u_{5}, u_{6}\right\}$ of $A$ such that $u_{1}^{2}=\alpha_{1} u_{5}+\alpha_{2} u_{6}, u_{1} u_{2}=$ $\alpha_{3} u_{5}+\alpha_{4} u_{6}, u_{1} u_{4}=\alpha_{5} u_{5}+\alpha_{6} u_{6}, u_{2}^{2}=\alpha_{7} u_{5}+\alpha_{8} u_{6}, u_{2} u_{4}=\alpha_{9} u_{5}+\alpha_{10} u_{6}$, $u_{3}^{2}=u_{5}, u_{3} u_{4}=u_{6}, u_{4}^{2}=\varepsilon u_{5}$, and other products zero.

Proof. It is possible to prove that there exist elements $y, x$ in $A$ such that $x, y, x^{2}, y x$ are linearly independent, and $y^{2}=\varepsilon x^{2}$ (see, [4]). We consider $u, v \in A$ such that $\left\{u, v, x, y, x^{2}, y x\right\}$ is a basis of $A$. Since $u x$ and $v x$ are elements in $A^{2}$, then $u x=\alpha x^{2}+\beta x y$ and $v x=\gamma x^{2}+\delta x y$. If $u_{0}=u-\alpha x-\beta y$ and $v_{0}=v-\gamma x-\delta y$, then $\left\{u_{0}, v_{0}, x, y, x^{2}, y x\right\}$ is a basis of $A$ with $u_{0} x=v_{0} x=0$. If we define $u_{1}=u_{0}, u_{2}=v_{0}, u_{3}=x, u_{4}=y$, 
$u_{5}=x^{2}, u_{6}=y x$, we obtain that $u_{1}^{2}=\alpha_{1} u_{5}+\alpha_{2} u_{6}, u_{1} u_{2}=\alpha_{3} u_{5}+\alpha_{4} u_{6}$, $u_{1} u_{4}=\alpha_{5} u_{5}+\alpha_{6} u_{6}, u_{2}^{2}=\alpha_{7} u_{5}+\alpha_{8} u_{6}, u_{2} u_{4}=\alpha_{9} u_{5}+\alpha_{10} u_{6}, u_{3}^{2}=u_{5}$, $u_{3} u_{4}=u_{6}, u_{4}^{2}=\varepsilon u_{5}$, and other products zero.

Proposition 2.9 If $\operatorname{dim}_{K}(A)=6, A^{3}=0$ and $\operatorname{dim}_{K}\left(A^{2}\right)=1$, then there exists a basis $\left\{u_{1}, u_{2}, u_{3}, u_{4}, u_{5}, u_{6}\right\}$ such that $u_{1}^{2}=u_{6}, u_{2}^{2}=\beta u_{6}, u_{3}^{2}=\gamma u_{6}$, $u_{4}^{2}=\delta u_{6}, u_{5}^{2}=\varepsilon u_{6}$, all other products being zero.

Proof. There is an element $u_{1}$ in $A$ such that $u_{1}^{2} \neq 0$, and so $A^{2}=<u_{1}^{2}>_{K}$. We can write $A$ as a direct sum $A=K u_{1}^{2} \oplus A_{0}$, where $A_{0}=K u_{1} \oplus W$ for some subespace $W$. The map $f: A_{0} \times A_{0} \rightarrow K$ defined by $x y=f(x, y) u_{1}^{2}$ for all $x, y$ in $A_{0}$ is a symmetric bilinear form. It is known that there is a basis $\left\{u_{1}, u_{2}, u_{3}, u_{4}, u_{5}\right\}$ of $A_{0}$ such that $f\left(u_{i}, u_{j}\right)=0$, if $i \neq j$. Finally, if $u_{6}=u_{1}^{2}$ we have that $\left\{u_{1}, u_{2}, u_{3}, u_{4}, u_{5}, u_{6}\right\}$ is a basis of $A$ such that $u_{1}^{2}=u_{6}, u_{2}^{2}=\beta u_{6}, u_{3}^{2}=\gamma u_{6}, u_{4}^{2}=\delta u_{6}, u_{5}^{2}=\varepsilon u_{6}$, all other products being zero.

\section{JORDAN NILALGEBRAS OF NILINDEX 4 AND DI- MENSION 6}

In this section, $A$ is a Jordan nilalgebra of nilindex 4 and dimension 6 . Therefore the identities $x^{2}(y x)=\left(x^{2} y\right) x$ and $x^{4}=\left(x^{2}\right) 2=0$ are valid in $A$. By linearization we obtain that also are valid in $A$ the following identities:

$$
\begin{gathered}
x^{2} y^{2}+2(x y)^{2}=0 \\
x^{2}(y x)=\left(x^{2} y\right) x=0
\end{gathered}
$$

In [3], we prove that any Jordan nilalgebra of nilindex $n \geq 4$ and dimension $k$ with $n+1 \leq k \leq n+2$, is nilpotent of index $n$. From this we conclude that $A^{4}=0$.

Proposition 3.1 If $\left(A^{2}\right) 2 \neq 0$, then there exists a basis $\left\{u_{1}, u_{2}, u_{3}, u_{4}, u_{5}, u_{6}\right\}$ of $A$ such that $u_{1}^{2}=u_{3}, u_{2}^{2}=u_{4}, u_{6}^{2}=-\frac{1}{2} u_{5}, u_{1} u_{2}=u_{6}, u_{3} u_{4}=u_{5}$, all other products being zero.

Proof. Since $\left(A^{2}\right)^{2} \neq 0$, there exist $x, y \in A$ such that $x^{2} y^{2} \neq 0$. We know that $2(x y)^{2}=-x^{2} y^{2} \neq 0, A^{4}=0$ and moreover $A\left(A^{2}\right)^{2} \subset A A^{3}=A^{4}=0$. We will prove that $x, y, x^{2}, y^{2}, x^{2} y^{2}, x y$ are linearly independent. It is easy to prove that $x^{2}, y^{2}, x^{2} y^{2}, x y$ are linearly independent. Now if $\alpha x+\beta y+\gamma x^{2}+\delta y^{2}+\varepsilon x^{2} y^{2}+\theta x y=0$, then $\alpha x+\beta y=-\left(\gamma x^{2}+\delta y^{2}+\right.$ 
$\left.\varepsilon x^{2} y^{2}+\theta x y\right)$ which implies $\alpha^{2} x^{2}+2 \alpha \beta x y+\beta^{2} y^{2}=\theta^{2}(x y)^{2}+2 \gamma \delta x^{2} y^{2}=$ $\left(-\frac{1}{2} \theta^{2}+2 \gamma \delta\right) x^{2} y^{2}$. Thus we conclude that $\alpha=\beta=0$, and clearly $\gamma=\delta=$ $\varepsilon=\theta=0$. Therefore $\left\{x, y, x^{2}, y^{2}, x^{2} y^{2}, x y\right\}$ is a basis of $A$, and moreover Proposition 1.1 implies that $A^{2}=<x^{2}, y^{2}, x^{2} y^{2}, x y>_{K}$. Now we will prove that $A^{3}=<x^{2} y^{2}>_{K}$. If $z \in A^{3}$, then $z=\gamma_{1} x^{2}+\delta_{1} y^{2}+\varepsilon_{1} x^{2} y^{2}+\theta_{1} x y$. So $0=x^{2} z=\delta_{1} x^{2} y^{2}$ implies $\delta_{1}=0,0=y^{2} z=\gamma_{1} x^{2} y^{2}$ implies $\gamma_{1}=0$, and $0=$ $(x y) z=\theta_{1}(x y)^{2}=-\frac{1}{2} \theta_{1} x^{2} y^{2}$ implies $\theta_{1}=0$. Hence $z=\varepsilon_{1} x^{2} y^{2}$, and thus $A^{3}=<x^{2} y^{2}>_{K}$. Therefore $y x^{2}=\delta_{0} x^{2} y^{2}, x y^{2}=\delta x^{2} y^{2}, x(x y)=\gamma(x y)^{2}$, $y(x y)=\gamma_{0}(x y)^{2}, x^{3}=\alpha x^{2} y^{2}, y^{3}=\alpha_{0} x^{2} y^{2}$. If $x_{0}=x-\delta x^{2}-\gamma x y-\alpha y^{2}$, $y_{0}=y-\delta_{0} y^{2}-\gamma_{0} x y-\alpha_{0} x^{2}$ and we define $u_{1}=x_{0}, u_{2}=y_{0}, u_{3}=x_{0}^{2}$, $u_{4}=y_{0}^{2}, u_{5}=x_{0}^{2} y_{0}^{2}, u_{6}=x_{0} y_{0}$, then we get that $\left\{u_{1}, u_{2}, u_{3}, u_{4}, u_{5}, u_{6}\right\}$ is a basis of $A$ such that $u_{1}^{2}=u_{3}, u_{2}^{2}=u_{4}, u_{6}^{2}=-\frac{1}{2} u_{5}, u_{1} u_{2}=u_{6}, u_{3} u_{4}=u_{5}$, all other products being zero.

Lemma $3.21 \leq \operatorname{dim}_{K}\left(A^{3}\right) \leq 2$

Proof. Since $2 \leq \operatorname{dim}_{K}\left(A^{2}\right) \leq 4$, then $1 \leq \operatorname{dim}_{K}\left(A^{3}\right) \leq 3$. Suppose that $\operatorname{dim}_{K}\left(A^{3}\right)=3$. Then there exist elements $y, z, u, v, x$ in $A$ such that $A^{3}=<$ $u y^{2}, v z^{2}, x^{3}>_{K}$. Clearly $x^{2} \notin A^{3}$, and so $A^{2}=<x^{2}, u y^{2}, v z^{2}, x^{3}>_{K}$. Hence $y^{2}=\alpha x^{2}+\beta u y^{2}+\gamma v z^{2}+\delta x^{3}$ and $z^{2}=\alpha_{0} x^{2}+\beta_{0} u y^{2}+\gamma_{0} v z^{2}+\delta_{0} x^{3}$. Since $A^{4}=0$, we obtain $u y^{2}=\alpha u x^{2}$ and $v z^{2}=\alpha_{0} v x^{2}$ with $\alpha \neq 0$ y $\alpha_{0} \neq 0$. Therefore $A^{2}=<x^{2}, u x^{2}, v x^{2}, x^{3}>_{K}$. Now it is easy to prove that $u, v$, $x, x^{2}, u x^{2}, v x^{2}, x^{3}$ are linearly independent, a contradiction. Therefore $1 \leq \operatorname{dim}_{K}\left(A^{3}\right) \leq 2$, as desired.

By Proposition 3.1 we know that there is a unique nilalgebra such that $\left(A^{2}\right)^{2} \neq 0$. In the following, we assume that $\left(A^{2}\right)^{2}=0$.

Proposition 3.3 Suppose that $\operatorname{dim}_{K}\left(A^{2}\right)=4$ and $\operatorname{dim}_{K}\left(A^{3}\right)=2$.

(a) If for all $y, x \in A$ we have that $y x^{2}, x^{3}$ are linearly dependent, then there exists a basis $\left\{u_{1}, u_{2}, u_{3}, u_{4}, u_{5}, u_{6}\right\}$ of $A$ such that $u_{1}^{2}=u_{2}$, $u_{1} u_{2}=u_{3}, u_{1} u_{4}=\gamma u_{2}+\delta u_{3}+\varepsilon u_{5}+\theta u_{6}, u_{1} u_{5}=u_{6}, u^{2} u_{4}=u_{3}$, $u_{4}^{2}=u_{5}, u_{4} u_{5}=u_{6}$, all other products being zero.

(b) If there exist elements $y, x$ in $A$ such that $y x^{2}, x^{3}$ are linearly independent, then there exists a basis $\left\{u_{1}, u_{2}, u_{3}, u_{4}, u_{5}, u_{6}\right\}$ of $A$ such that $u_{1}^{2}=u_{6}, u_{1} u_{2}=\alpha u_{3}+\beta u_{5}+\gamma u_{6}, u_{1} u_{3}=u_{4}, u_{1} u_{6}=\delta u_{4}+\varepsilon u_{5}$, $u_{2}^{2}=u_{3}, u_{2} u_{3}=u_{5}, u_{2} u_{6}=\theta u_{4}+\sigma u_{5}$, all other products being zero. 
Proof. To prove $(a)$, we consider an element $x \in A$ with $x^{3} \neq 0$. By hypothesis, we have that for all $y \in A: y x^{2} \in<x^{3}>_{K}$. As $A^{4}=0$, we have that $J=<x^{2}, x^{3}>_{K}$ is an ideal of $A$, and moreover $A^{3}$ is not a subset of $J$. Now if $y^{3} \in J$ for all $y \in A$, then the quotient algebra $\bar{A}=A / J$ is a nilalgebra of nilindex 3 with $\operatorname{dim}_{K}(\bar{A})=4$ and $\bar{A}^{3} \neq \overline{0}$ which is a contradiction, since by Lemma 2.4 we know that $\operatorname{dim}_{K}(\bar{A}) \geq 5$. Therefore there exists $y \in A$ such that $y^{3} \notin<x^{2}, x^{3}>_{K}$. By hypothesis $y x^{2}=\alpha x^{3}$, $x y^{2}=\beta y^{3}$. Now it is possible to prove that $x, y, x^{2}, x^{3}, y^{2}, y^{3}$ are linearly independent, and so $x y=\gamma_{0} x^{2}+\delta_{0} x^{3}+\varepsilon_{0} y^{2}+\theta_{0} y^{3}$. By hypothesis for all $\gamma_{1}, \delta_{1}, \alpha_{1}, \beta_{1}$ in $K$, we have that the vectors $\left(\gamma_{1} x+\delta_{1} y\right)^{3},\left(\alpha_{1} x+\right.$ $\left.\beta_{1} y\right)\left(\gamma_{1} x+\delta_{1} y\right)^{2}$ are linearly dependent. Now we have that $\left(\gamma_{1} x+\delta_{1} y\right)^{3}=$ $\left(\gamma_{1}^{3}+2 \gamma_{1}^{2} \delta_{1} \gamma_{0}+\gamma_{1}^{2} \delta_{1} \alpha+2 \gamma_{1} \delta_{1}^{2} \gamma_{0} \alpha\right) x^{3}+\left(\gamma_{1} \delta_{1}^{2} \beta+2 \gamma_{1}^{2} \delta_{1} \beta \varepsilon_{0}+\delta_{1}^{3}+2 \gamma_{1} \delta_{1}^{2} \varepsilon_{0}\right) y^{3}$ and $\left(\alpha_{1} x+\beta_{1} y\right)\left(\gamma_{1} x+\delta_{1} y\right)^{2}=\left(\gamma_{1}^{2} \alpha_{1}+2 \gamma_{1} \alpha_{1} \delta_{1} \gamma_{0}+\gamma_{1}^{2} \beta_{1} \alpha+2 \gamma_{1} \delta_{1} \beta_{1} \alpha \gamma_{0}\right) x^{3}+$ $\left(\delta_{1}^{2} \alpha_{1} \beta+2 \gamma_{1} \alpha_{1} \delta_{1} \beta \varepsilon_{0}+\delta_{1}^{2} \beta_{1}+2 \gamma_{1} \delta_{1} \beta_{1} \varepsilon_{0}\right) y^{3}$. We conclude that for all $\gamma_{1}$, $\delta_{1}, \alpha_{1}, \beta_{1}$ in $K$ the vectors $\left(\gamma_{1}^{3}+2 \gamma_{1}^{2} \delta_{1} \gamma_{0}+\gamma_{1}^{2} \delta_{1} \alpha+2 \gamma_{1} \delta_{1}^{2} \gamma_{0} \alpha, \gamma_{1} \delta_{1}^{2} \beta+\right.$ $\left.2 \gamma_{1}^{2} \delta_{1} \beta \varepsilon_{0}+\delta_{1}^{3}+2 \gamma_{1} \delta_{1}^{2} \varepsilon_{0}\right)$ and $\left(\gamma_{1}^{2} \alpha_{1}+2 \gamma_{1} \alpha_{1} \delta_{1} \gamma_{0}+\gamma_{1}^{2} \beta_{1} \alpha+2 \gamma_{1} \delta_{1} \beta_{1} \alpha \gamma_{0}\right.$, $\left.\delta_{1}^{2} \alpha_{1} \beta+2 \gamma_{1} \alpha_{1} \delta_{1} \beta \varepsilon_{0}+\delta_{1}^{2} \beta_{1}+2 \gamma_{1} \delta_{1} \beta_{1} \varepsilon_{0}\right)$ in $K^{2}$ are linearly dependent, which implies that $\alpha \beta=1$. Finally, if $u_{1}=x, u_{2}=x^{2}, u_{3}=x^{3}, u_{4}=\beta y$, $u_{5}=\beta^{2} y^{2}, u_{6}=\beta^{3} y^{3}$, we obtain $(a)$. To prove $(b)$, we consider $y, x \in A$ such that $y x^{2}, x^{3}$ are linearly independent. Then $A^{3}=<y x^{2}, x^{3}>_{K}$ and $x^{2}$, $y x^{2}, x^{3}$ are linearly independent. As $\operatorname{dim}_{K}\left(A^{2}\right)=4$, there exists $z \in A$ such that $A^{2}=<x^{2}, y x^{2}, x^{3}, z^{2}>_{K}$. It is easy to prove that $\left\{y, x, x^{2}, y x^{2}, x^{3}, z^{2}\right\}$ is a basis of $A$. Now if $z=\alpha_{1} y+\alpha_{2} x+\alpha_{3} x^{2}+\alpha_{4} y x^{2}+\alpha_{5} x^{3}+\alpha_{6} z^{2}$, then $z^{2}-\left(\alpha_{1}^{2} y^{2}+2 \alpha_{1} \alpha_{2} x y+\alpha_{2}^{2} x^{2}\right) \in A^{3}$ which implies $\alpha_{1} \neq 0$. If $y_{0}=$ $\alpha_{1} y+\alpha_{2} x$, then $y_{0}^{2} \notin<x^{2}, y x^{2}, x^{3}>_{K}=<x^{2}, y_{0} x^{2}, x^{3}>_{K}$, and so $A^{2}=<$ $x^{2}, y_{0} x^{2}, x^{3}, y_{0}^{2}>$. If $y_{0} x=\alpha x^{2}+\lambda y_{0} x^{2}+\beta x^{3}+\gamma y_{0}^{2}$ and $x_{0}=x-\lambda x^{2}$, then $y_{0} x_{0}=\alpha x^{2}+\beta x^{3}+\gamma y_{0}^{2} \in<x_{0}^{2}, x_{0}^{3}, y_{0}^{2}>_{K}$. Therefore we can assume that there exist elements $y, x$ in $A$ such that $\left\{y, x, x^{2}, y x^{2}, x^{3}, y^{2}\right\}$ is a basis of $A$ with $y x=\alpha x^{2}+\beta x^{3}+\gamma y^{2}, y^{3}=\delta y x^{2}+\varepsilon x^{3}$ and $x y^{2}=\theta y x^{2}+\sigma x^{3}$. If we define $u_{1}=y, u_{2}=x, u_{3}=x^{2}, u_{4}=y x^{2}, u_{5}=x^{3}, u_{6}=y^{2}$, we obtain (b).

Proposition 3.4 If $\operatorname{dim}_{K}\left(A^{2}\right)=4$ and $\operatorname{dim}_{K}\left(A^{3}\right)=1$, then there exists a basis $\left\{u_{1}, u_{2}, u_{3}, u_{4}, u_{5}, u_{6}\right\}$ of $A$ such that $u_{1}^{2}=u_{5}, u_{1} u_{2}=u_{6}, u_{1} u_{5}=\beta u_{4}$, $u_{1} u_{6}=\gamma u_{4}, u_{2}^{2}=u_{3}, u_{2} u_{3}=u_{4}, u_{2} u_{5}=\delta u_{4}, u_{2} u_{6}=\varepsilon u_{4}$, all other products being zero.

Proof. We consider $x \in A$ such that $x^{3} \neq 0$. Since $\operatorname{dim}_{K}\left(A^{2}\right)=4$, there are $y, z \in A$ such that $A^{2}=<x^{2}, x^{3}, y^{2}, z^{2}>_{K}$. We have that $y, x, x^{2}$, $x^{3}, y^{2}, z^{2}$ are linearly independent. In fact: if $\alpha_{1} y+\alpha_{2} x+\alpha_{3} x^{2}+\alpha_{4} x^{3}+$ 
$\alpha_{5} y^{2}+\alpha_{6} z^{2}=0$, then $\alpha_{1} y=-\left(\alpha_{2} x+\alpha_{3} x^{2}+\alpha_{4} x^{3}+\alpha_{5} y^{2}+\alpha_{6} z^{2}\right)$ which implies $\alpha_{1}^{2} y^{2}=\alpha_{2}^{2} x^{2}+v$ with $v \in A^{3}=<x^{3}>_{K}$. Hence $\alpha_{1}=\alpha_{2}=0$, and so $\left\{y, x, x^{2}, x^{3}, y^{2}, z^{2}\right\}$ is a basis of $A$. If $z=\beta_{1} y+\beta_{2} x+\beta_{3} x^{2}+\beta_{4} x^{3}+$ $\beta_{5} y^{2}+\beta_{6} z^{2}$, then $z^{2}-\left(\beta_{1}^{2} y^{2}+2 \beta_{1} \beta_{2} y x+\beta_{2}^{2} x^{2}\right) \in A^{3}$ which implies that $y x \notin<x^{2}, x^{3}, y^{2}>_{K}$, and therefore $A^{2}=<x^{2}, x^{3}, y^{2}, y x>_{K}$. We see that as $y x^{2}=\alpha x^{3}$, then $x^{2}\left(y-\alpha x^{2}\right)=0$. Therefore we can assume that in the basis $\left\{y, x, x^{2}, x^{3}, y^{2}, y x\right\}$ of $A$ we have that $y x^{2}=0$, and moreover $y^{3}=\beta x^{3}, y(y x)=\gamma x^{3}, x y^{2}=\delta x^{3}, x(y x)=\varepsilon x^{3}$. Finally, if we define $u_{1}=y, u_{2}=x, u_{3}=x^{2}, u_{4}=x^{3}, u_{5}=y^{2}, u_{6}=y x$, we obtain our Proposition.

Proposition 3.5 If $\operatorname{dim}_{K}\left(A^{2}\right)=3$ and $\operatorname{dim}_{K}\left(A^{3}\right)=2$, then there exists a basis $\left\{u_{1}, u_{2}, u_{3}, u_{4}, u_{5}, u_{6}\right\}$ of $A$ sucha that $u_{1}^{2}=\gamma_{1} u_{4}+\gamma_{2} u_{5}+\gamma_{3} u_{6}$, $u_{1} u_{2}=\delta_{1} u_{4}+\delta_{1} u_{4}+\delta_{2} u_{5}+\delta_{3} u_{6}, u_{1} u_{3}=\beta u_{5}, u_{1} u_{4}=\lambda_{1} u_{5}+\lambda_{2} u_{6}$, $u_{2}^{2}=\varepsilon_{1} u_{4}+\varepsilon_{2} u_{5}+\varepsilon_{3} u_{6}, u_{2} u_{4}=u_{5}, u_{3}^{2}=u_{4}, u_{3} u_{4}=u_{6}$, all other products being zero.

Proof. By Proposition 3.1, it is clear that $\left(A^{2}\right)^{2}=0$. We consider $x \in A$ such that $x^{3} \neq 0$. We note that if $I=<x^{2}, x^{3}>_{K}$ is an ideal of $A$, then $y x^{2} \in<x^{3}>_{K}$ for all $y \in A$, and $A^{3}$ is not a subset of $I$. If $I$ is an ideal of $A$ and $z^{3} \in I$ for all $z \in A$, then the quotient algebra $\bar{A}=A / I$ is of nilindex 3 with $\bar{A}^{3}=\overline{0}$, which implies $\operatorname{dim}_{K}\left(\bar{A}^{3}\right) \geq 5$, a contradiction. Hence if $I$ is an ideal of $A$, then there is $y \in A$ such that $y^{3} \notin I$, and so $A^{2}=<$ $x^{2}, x^{3}, y^{3}>_{K}$. Since $y^{2} \in A^{2}=<x^{2}, x^{3}, y^{3}>_{K}$, then $y^{3} \in<y x^{2}>_{K} \subset<$ $x^{3}>_{K}$, a contradiction. Therefore we conclude that $I=<x^{2}, x^{3}>_{K}$ is not an ideal of $A$ and so there exists an element $y \in A$ such that $y x^{2}$, $x^{3}$ are linearly independent. In this case it is possible to prove that $y, x$, $x^{2}, y x^{2}, x^{3}$ are linearly independent, and thus $A^{2}=<x^{2}, y x^{2}, x^{3}>_{K}$ and $A^{3}=<y x^{2}, x^{3}>_{K}$. If $y x=\beta_{1} x^{2}+\beta_{2} y x^{2}+\beta_{3} x^{3}$, then $y x_{0}=\beta_{1} x^{2}+\beta_{3} x^{3} \in<$ $x_{0}^{2}, x_{0}^{3}>_{K}$ where $x_{0}=x-\beta_{2} x^{2}$. Thus we can suppose that $y x=\beta_{1} x^{2}+\beta_{3} x^{3}$, which implies $y_{0} x=0$ where $y_{0}=y-\beta_{1} x-\beta_{3} x^{2}$. Therefore we can assume that $y, x, x^{2}, y x^{2}, x^{3}$ are linearly independent with $y x=0$. Now it is easy to find an element $z \in A$ such that $\left\{z, y, x, x^{2}, y x^{2}, x^{3}\right\}$ is a basis of $A$ with $x z=\beta y x^{2}$. Moreover we have that $z^{2}=\gamma_{1} x^{2}+\gamma_{2} y x^{2}+\gamma_{3} x^{3}$, $y z=\delta_{1} x^{2}+\delta_{2} y x^{2}+\delta_{3} x^{3}, y^{2}=\varepsilon_{1} x^{2}+\varepsilon_{2} y x^{2}+\varepsilon_{3} x^{3}, z x^{2}=\lambda_{1} y x^{2}+\lambda_{2} x^{3}$. If we define $u_{1}=z, u_{2}=y, u_{3}=x, u_{4}=x^{2}, u_{5}=y x^{2}, u_{6}=x^{3}$, we obtain our Proposition.

Proposition 3.6 If $\operatorname{dim}_{K}\left(A^{2}\right)=3$ and $\operatorname{dim}_{K}\left(A^{3}\right)=1$, then there exists a basis $\left\{u_{1}, u_{2}, u_{3}, u_{4}, u_{5}, u_{6}\right\}$ of $A$ such that $u_{1}^{2}=\alpha_{1} u_{4}+\alpha_{2} u_{5}+\alpha_{3} u_{6}$, 
$u_{1} u_{2}=\beta_{1} u_{4}+\beta_{2} u_{5}+\beta_{3} u_{6}, u_{1} u_{3}=\gamma u_{6}, u_{1} u_{4}=\delta_{0} u_{5}, u_{1} u_{6}=\lambda u_{5}, u_{2}^{2}=u_{6}$, $u_{2} u_{3}=\gamma_{1} u_{4}+\gamma_{2} u_{5}+\gamma_{3} u_{6}, u_{2} u_{4}=\delta u_{5}, u_{2} u_{6}=\varepsilon u_{5}, u_{3}^{2}=u_{4}, u_{3} u_{4}=u_{5}$, $u_{3} u_{6}=\theta u_{5}$, all other products being zero.

Proof. Clearly $\left(A^{2}\right)^{2}=0$. We consider $x \in A$ with $x^{3} \neq 0$. Then $A^{3}=<x^{3}>_{K}$ and there is $y \in A$ such that $A^{2}=<x^{2}, x^{3}, y^{2}>_{K}$. It is easy to show that $y, x, x^{2}, x^{3}, y^{2}$ are linearly independent. It is easy to find an element $z \in A$ such that $\left\{z, y, x, x^{2}, x^{3}, y^{2}\right\}$ is a basis of $A$ with $z x=\gamma y^{2}$. If we define $u_{1}=z, u_{2}=y, u_{3}=x, u_{4}=x^{2}, u_{5}=x^{3}, u_{6}=y^{2}$, we obtain our Proposition.

Proposition 3.7 If $\operatorname{dim}_{K}\left(A^{2}\right)=2$ and $\operatorname{dim}_{K}\left(A^{3}\right)=1$, then there exists a basis $\left\{u_{1}, u_{2}, u_{3}, u_{4}, u_{5}, u_{6}\right\}$ of $A$ such that $u_{1}^{2}=\alpha_{1} u_{5}+\alpha_{2} u_{6}, u_{1} u_{2}=\beta_{1} u_{5}+$ $\beta_{2} u_{6}, u_{1} u_{3}=\gamma_{1} u_{5}+\gamma_{2} u_{6}, u_{1} u_{5}=\alpha u_{6}, u_{2}^{2}=\delta_{1} u_{5}+\delta_{2} u_{6}, u_{2} u_{3}=\varepsilon_{1} u_{5}+\varepsilon_{2} u_{6}$, $u_{2} u_{5}=\beta u_{6}, u_{3}^{2}=\lambda_{1} u_{5}+\lambda_{2} u_{6}, u_{3} u_{5}=\gamma u_{6}, u_{4}^{2}=u_{5}, u_{4} u_{5}=u_{6}$, all other products being zero.

Proof. We consider $x \in A$ with $x^{3} \neq 0$. Then $A^{3}=<x^{3}>_{K}$ and $A^{2}=<$ $x^{2}, x^{3}>_{K}$. It is easy find elements $y, z, v$ in $A$ such that $\left\{y, z, v, x, x^{2}, x^{3}\right\}$ is a basis of $A$ with $y x=z x=v x=0$. Now we have that $y^{2}=\alpha_{1} x^{2}+\alpha_{2} x^{3}$, $y z=\beta_{1} x^{2}+\beta_{2} x^{3}, y v=\gamma_{1} x^{2}+\gamma_{2} x^{3}, y x^{2}=\alpha x^{3}, z^{2}=\delta_{1} x^{2}+\delta_{2} x^{3}$, $z v=\varepsilon_{1} x^{2}+\varepsilon_{2} x^{3}, z x^{2}=\beta x^{3}, v^{2}=\lambda_{1} x^{2}+\lambda_{2} x^{3}, v x^{2}=\gamma x^{3}$. Finally, if $u_{1}=y, u_{2}=z, u_{3}=v, u_{4}=x, u_{5}=x^{2}, u_{6}=x^{3}$, we obtain our Proposition.

\section{JORDAN NILALGEBRAS OF NILINDEX k AND DI- MENSION 6 WITH $\mathrm{k} \geq 5$}

In [2], we describe Jordan nilalgebras of nilindex $n$ and dimension $n+1$. In this work, we find the following results:

Proposition 4.1 If $A$ is a Jordan nilalgebra of nilindex 5 and dimension $6, \operatorname{dim}_{K}\left(A^{2}\right)=4$ and $\operatorname{dim}_{K}\left(A^{3}\right)=2$, then there exists a basis $\left\{u_{1}, u_{2}, u_{3}, u_{4}, u_{5}, u_{6}\right\}$ of $A$ such that $u_{1}^{2}=\alpha u_{2}+\gamma_{2} u_{4}+\gamma_{3} u_{5}+\gamma_{4} u_{6}, u_{1} u_{2}=$ $\beta_{0} u_{5}+\gamma_{0} u_{6}, u_{1} u_{3}=u_{2}, u_{1} u_{5}=-2 \beta u_{6}, 2 u_{2}^{2}=\beta(\alpha-4 \beta) u_{6}, u_{2} u_{3}=$ $\beta u_{5}+\gamma u_{6}, u_{3}^{2}=u_{4}, u_{3} u_{4}=u_{5}, u_{3} u_{5}=u_{6}, u_{4}^{2}=u_{6}$, all other products being zero. Moreover, if $\beta=0$ then $\gamma_{2}=\beta_{0}=0$, if $\beta \neq 0$ and $\alpha=4 \beta$ then $\gamma_{2}=-4 \beta^{2}, \beta_{0}=-2 \beta^{2}$, if $\beta \neq 0$ and $\alpha \neq 4 \beta$ then $\alpha=-4 \beta$, $\gamma_{2}=-4 \beta^{2}$ and $\beta_{0}=-6 \beta^{2}$. 
Proposition 4.2 If $A$ is a Jordan nilalgebra of nilindex 5 and dimension $6, \operatorname{dim}_{K}\left(A^{2}\right)=4$ and $\operatorname{dim}_{K}\left(A^{3}\right)=3$, then there exists a basis $\left\{u_{1}, u_{2}, u_{3}, u_{4}, u_{5}, u_{6}\right\}$ of $A$ such that $u_{1} u_{4}=u_{2}, u_{1}^{2}=\lambda u_{2}+\delta u_{4}+\gamma u_{5}+\varepsilon u_{6}$, $u_{1} u_{2}=\delta u_{6}, u_{3}^{2}=u_{4}, u_{3} u_{4}=u_{5}, u_{3} u_{5}=u_{6}, u_{4}^{2}=u_{6}$, all other products zero.

Proposition 4.3 If $A$ is a Jordan nilalgebra of nilindex 5 and dimension 6 and $\operatorname{dim}_{K}\left(A^{2}\right)=3$, and then there exists a basis $\left\{u_{1}, u_{2}, u_{3}, u_{4}, u_{5}, u_{6}\right\}$ of $A$ such that $u_{1} u_{3}=\alpha u_{5}, u_{1}^{2}=\beta u_{5}+\gamma u_{6}, u_{2} u_{3}=\alpha_{0} u_{5}, u_{2}^{2}=\beta_{0} u_{5}+\gamma_{0} u_{6}$, $u_{1} u_{2}=\delta u_{5}+\varepsilon u_{6}, u_{3}^{2}=u_{4}, u_{3} u_{4}=u_{5}, u_{3} u_{5}=u_{6}, u_{4}^{2}=u_{6}$, all other products being zero.

In [1], the authors proved the following result:

Proposition 4.4 If $A$ is a Jordan nilalgebra of nilindex 6 and dimension 6, then there exists a basis $\left\{u_{1}, u_{2}, u_{3}, u_{4}, u_{5}, u_{6}\right\}$ of $A$ such that $u_{1}^{2}=\beta u_{5}+$ $\gamma u_{6}, u_{1} u_{2}=\alpha u_{5}, u_{2}^{2}=u_{3}, u_{2} u_{3}=u_{4}, u_{2} u_{4}=u_{5}, u_{2} u_{5}=u_{6}, u_{3}^{2}=u_{5}$, $u_{3} u_{4}=u_{6}$, all other products zero.

Moreover in this case it is possible to find five classes of algebras which are not isomorphic (see [1], Theorem 3).

Remark Finally, it is clear that there is a unique Jordan nilalgebra of nilindex 7 and dimension 6 .

\section{REFERENCES}

[1] I. Correa and A. Suazo, On a class of commutative power-associative nilalgebras, Journal of Algebra 215, pp. 412-417, (1999).

[2] L. Elgueta and A. Suazo, Jordan nilagebras of nilindex $n$ and dimension $n+1$, Communications in Algebra, Vol. 30, 11, pp. 5545-5559, (2002).

[3] L. Elgueta and A. Suazo, The index of nilpotence of Jordan nilalgebras of nilindex $n$ and dimension $\leq n+2$, International Journal of Mathematics, Game Theory and Algebra, to appear (2002).

[4] M. Gerstenhaber and H. C. Myung, On commutative power-associative nilalgebras of low dimension, Proc. Amer. Math. Soc. 48, pp. 29-32, (1975). 
[5] R. D. Schafer, An Introduction to Nonassociative Algebras, Academic Press, New York/London, (1966).

[6] K. A. Zhevlakov, A. M. Slin'ko, I. P. Shestakov, and A. I. Shirshov, Rings That Are Nearly Associative, Academic Press, New York/London, (1992).

Received : June, 2002

\section{Luisa Elgueta}

Departamento de Matemáticas

Universidad de La Serena

Benavente 980

La Serena

Chile

e-mail : lelgueta@userena.cl

and

\section{Avelino Suazo}

Departamento de Matemáticas

Universidad de La Serena

Benavente 980

La Serena

Chile

e-mail : asuazo@userena.cl 